\title{
LA INVESTIGACIÓN SOBRE GERONTOLOGÍA EDUCATIVA EN ESPAÑA: ANÁLISIS A TRAVÉS DE LAS REVISTAS ESPAÑOLAS DE EDUCACIÓN INCLUIDAS EN EL JCR
}

\author{
M. R. BELANDO-MONTORO \\ Universidad Complutense de Madrid (España) \\ mbelando@ucm.es
}

Artículo presentado en junio/2016 y aceptado en julio/2016

DOI: $10.15628 /$ holos. 2016.4730

\section{RESUMEN}

Este trabajo es un análisis de los artículos sobre la gerontología educativa publicados en las revistas educativas españolas (incluidas en el Journal Citation Report), con el fin de proporcionar una visión diacrónica de la productividad científica de esta área entre 2000 y 2014 y describir una visión general de la gerontología educativa a través de análisis cuantitativo y cualitativo de un número de variables. Los resultados muestran que la bibliografía sobre gerontología educativa no se limita a un área geográfica específica, los autores muestran una notable variedad de intereses y hay una ligera diferencia a favor del trabajo realizado por las mujeres. Entre los puntos débiles son la baja producción, el bajo nivel de la multidisciplinariedad en los artículos, la ausencia de estudios longitudinales y los co-autores, hay poca colaboración institucional y geográfica.

PALABRAS CLAVE: Gerontología; Educación, Investigación, revistas de impacto, España.

\section{RESEARCH ON EDUCATIONAL GERONTOLOGY IN SPAIN: ANALYSIS THROUGH THE SPANISH EDUCATIONAL JOURNALS IN THE JCR}

\begin{abstract}
This paper analyses the articles on educational gerontology published in the impact Spanish journals (from JCR) in the field of the education with the aim to provide a diachronic view of scientific productivity of this area between 2000 and 2014 and describe an overview of educational gerontology through quantitative and qualitative analysis of a number of variables. The outcomes reveal that the literature on educational
\end{abstract}

gerontology are not bounded to a specific geographical area, the authors show a remarkable range of interests and there is a slight difference in favor of the articles done by women. Among the weaknesses highlight a production scarce, a low degree of multidisciplinarity in the articles, concerning co-authorship there are hardly any institutional and geographical collaboration and no longitudinal studies.

KEYWORDS: Gerontology, Education, Research, Impact Journals, Spain. 


\section{INTRODUCCIÓN}

El envejecimiento de la población es un fenómeno global y las tendencias demográficas indican que este fenómeno sigue un camino de no retorno. Si en 1950 había un 8\% de personas mayores de 60 años en el mundo, en el 2000 esta cifra aumentó a un 10\% y los cálculos para el 2050 son de un $22 \%$. Además, las previsiones, a nivel mundial, indican que el número de personas de edad superará por primera vez el de niños en 2045 (United Nations, 2010).

Ahora bien, hay diferencias significativas entre las diferentes regiones, siendo Europa y Japón los que muestran proporciones mucho mayores a las observadas en el resto del mundo (Schoenmaeckers, 2005; United Nations, 2010). De hecho, el envejecimiento de la población europea es uno de los desafíos sociales más importantes a los que se enfrenta esta región. De aquí las constantes exhortaciones procedentes desde las diversas instituciones de la Unión Europea realizadas en los últimos años cuyo objetivo fundamental es el envejecimiento activo y saludable. Ejemplo de ello es la declaración de 2012 como Año Europeo del Envejecimiento Activo, cuya finalidad era

\footnotetext{
"fomentar y apoyar los esfuerzos de los Estados miembros y sus autoridades regionales y locales, de los interlocutores sociales y de la sociedad civil para promover el envejecimiento activo y redoblar el esfuerzo por movilizar el potencial de las personas, cada vez más numerosas, próximas a los sesenta años o mayores, preservando con ello la solidaridad intergeneracional" (Comisión Europea, 2010, p. 10).
}

Esta preocupación de la Unión Europea es el reflejo del interés que la mayoría de los diversos Estados miembros están mostrando desde hace ya bastantes años. En el caso concreto de España, destaca su posición entre los veinticinco países más envejecidos del mundo y las previsiones para el año 2050 lo sitúan en el séptimo puesto (United Nations, 2013). Esta circunstancia, largamente advertida en las series históricas del Padrón del Instituto Nacional de Estadística, ha provocado diversas iniciativas desde finales de los ochenta de la pasada centuria, tanto desde el ámbito político a través de planes gerontológicos nacionales y autonómicos, como desde el privado. En este último ámbito se han ido desarrollando todo tipo de empresas dedicadas a la atención y al cuidado de los mayores.

En la actualidad, el envejecimiento de la población española está teniendo consecuencias en muchos y muy diversos campos. Especialmente, en el económico, donde suele adquirir un protagonismo significativo, sobre todo, en lo referente a las pensiones. Pero el fenómeno del envejecimiento también afecta al consumo, a la demanda de viviendas adaptadas a las necesidades de la población mayor, a la composición de las familias, a los servicios sanitarios y de ocio, a las tendencias migratorias y a la composición del voto.

Paralelamente al aumento de la población mayor en España -que en los últimos veinte años ha pasado de un 13,8\% a un 17,7\% (Instituto Nacional de Estadística 1995, 2014)-, ha ido creciendo la investigación española sobre el envejecimiento, hasta alcanzar el $1 \%$ de la producción científica española. Diversos organismos, principalmente el Ministerio de Economía y Competitividad (anteriormente el Ministerio de Ciencia e Innovación), el Instituto de Salud Carlos III y el Instituto de Mayores y Servicios Sociales (en adelante IMSERSO), financian investigaciones en los campos 
sanitario y social. Si bien, la mayor parte de los trabajos pertenecen al campo de la Biología y la Medicina y en una segunda posición se sitúan los de Psicología (Rodríguez, 2011). Para la Educación, este campo supone aún hoy un tema pendiente. De hecho, en las convocatorias del 2004 al 2011 del Subprograma de Proyectos de Investigación Fundamental No Orientada del MICINN, ninguno de los proyectos financiados se refería al ámbito de la gerontología educativa. Sólo en la convocatoria de 2012 encontramos un proyecto orientado al envejecimiento satisfactorio. En 2013 tampoco hubo ningún proyecto de este ámbito y en el 2014 no hubo convocatoria. Respecto a las resoluciones de las convocatorias del Instituto de Mayores y Servicios Sociales entre los años $2005^{1}$ y $2011^{2}$ para la subvención a proyectos de investigación científica, desarrollo e innovación tecnológica, los resultados son algo más positivos aunque siguen constituyendo una minoría respecto a otros ámbitos científicos. En los años 2005, 2006 y 2007 se concedieron 5 proyectos ${ }^{3}$.

Frente a ello, el papel de la educación en la calidad de vida de las personas mayores, así como en el envejecimiento activo, ha sido reconocido en multitud de ocasiones, y no sólo en las publicaciones específicas del área sino también en el contenido de documentos y declaraciones de organismos nacionales e internacionales. En el caso del IMSERSO, el documento de trabajo preparatorio para la elaboración del "Libro Blanco del Envejecimiento Activo", propone la educación a lo largo de la vida como uno de los nueve aspectos más relevantes del envejecimiento activo y se reconoce que ello permite avanzar en el ejercicio de los derechos de las personas de edad, les ayuda a su integración en una sociedad plural y global y favorece el proceso de construcción de una identidad personal positiva (IMSERSO, 2010).

En el ámbito europeo, las campañas de educación son una de las medidas establecidas para el logro de los objetivos del Año Europeo del Envejecimiento Activo (Comisión Europea, 2010). Posteriormente, en las recomendaciones del Comité de las Regiones al Consejo y a la Comisión Europea, se recordaba que el aprendizaje permanente es un factor clave del envejecimiento activo y saludable y se ponía de relieve que "la formación de adultos y el voluntariado de las personas mayores debería fomentarse a nivel europeo, nacional y local para ayudar a prolongar la vida laboral, favorecer la jubilación activa y contribuir a la vida independiente" (Comité de las Regiones 2012, p. 49). Atendiendo a lo recogido en el informe sobre la evaluación del Año Europeo del Envejecimiento Activo (Comisión Europea, 2014), el objetivo del aprendizaje mutuo sobre los temas del envejecimiento activo se logró especialmente a través de proyectos nacionales y trasnacionales.

También Naciones Unidas ha manifestado este interés. En el II Plan Internacional sobre el Envejecimiento, aprobado en la segunda Asamblea Mundial sobre el Envejecimiento celebrada en abril de 2002 en Madrid, se hace varias referencias a la importancia de la educación en esta etapa de la vida. Por un lado, se hace hincapié en la idea de la población de edad como fuerza productiva en beneficio de la sociedad. Esto se observa claramente en el enunciado de sus dos objetivos:

\footnotetext{
${ }^{1}$ No se disponen de datos previos al 2005.

2 En los años 2012, 2013 y 2014 no se convocaron subvenciones para la realización de proyectos de investigación.

${ }^{3}$ Uno en 2005, uno en 2006 y tres en 2007. Tres de ellos se centraban en la evaluación de los programas universitarios para mayores, otro proyecto también se refería a la formación de los mayores en el ámbito universitario y la finalidad del quinto era formar a los mayores como profesores de español.
} 
1. Igualdad de oportunidades durante toda la vida en materia de educación permanente, capacitación y readiestramiento, así como de orientación profesional y acceso a servicios de colocación laboral.

2. Utilización plena de las posibilidades y los conocimientos de las personas de todas las edades, reconociendo los beneficios derivados de la mayor experiencia adquirida con la edad.

Pero, además de la capacitación profesional, el Plan también recoge otras indicaciones referidas al ámbito educativo. El análisis de dichas alusiones permite realizar una distinción en cuatro bloques temáticos, de los cuales dos aluden a los beneficios de la educación de los propios mayores:

a) La educación en esta etapa de la vida favorece la realización personal, el bienestar y la participación en la vida cultural, política y social, entre otros.

b) El potencial educativo de los mayores debe ser reconocido, potenciado y aprovechado. En concreto, se habla de las personas de edad como líderes, tanto en su familia como en su comunidad, en materia de educación. Se desarrolla, asimismo, la importancia del intercambio de conocimiento y experiencia entre las generaciones.

Llegados a este punto, y habiendo realizado una revisión del reconocimiento que, desde diversos organismos nacionales e internacionales, se hace a la educación como elemento fundamental en la construcción de un envejecimiento activo -lo que no se refleja en los proyectos de investigación financiados en España-, es pertinente plantear si las publicaciones científicas españolas se han hecho eco del fenómeno del envejecimiento de la población y han contribuido a la construcción de un campo temático preocupado por la calidad de vida en la vejez, como es el caso de la gerontología educativa. Ahora bien, antes de centrar el análisis en el ámbito específico de interés de este estudio, es preciso realizar una panorámica acerca de las publicaciones científicas españolas que nos posibilite una visión más amplia sobre la que situar y comprender la situación de las publicaciones españolas que incluyen las cuestiones relativas a la educación y la vejez, en especial, las revistas españolas de educación de calidad contrastada.

\section{SOBRE LA CALIDAD EN LAS REVISTAS CIENTÍFICAS ESPAÑOLAS}

Como ha sido reconocido por diversos especialistas (Hernández y Maquilón, 2010), la falta de calidad de las publicaciones científicas españolas ha obstaculizado su impacto internacional. Sin embargo, en los últimos años la necesidad de incrementar el prestigio y el impacto de las revistas científicas en España, paralelamente a la incorporación de la nueva normativa para la evaluación de la investigación de los profesores universitarios y del personal científico (ANECA, CNEAI y las diversas Agencias Autonómicas de Evaluación de la Calidad Universitaria), ha provocado el surgimiento de notables iniciativas, entre las que cabe destacar el programa ARCE (Apoyo a las Revistas Científicas Españolas) de la Fundación Española para la Ciencia y la Tecnología (en adelante FECYT).

A través de dicho programa se pretende impulsar la profesionalización e internacionalización de las revistas científicas españolas de calidad contrastada. Las revistas que superan el proceso de evaluación obtienen un reconocimiento de la calidad editorial y científica 
por un periodo de dos años y el acceso a la herramienta de gestión editorial electrónica (Open Journal Systems-OJS) alojada en el Repositorio Español de Ciencia y Tecnología (RECYT).

La primera convocatoria para obtener el Sello de Calidad FECYT se realizó en 2007. Desde entonces, doce revistas específicas del área de Ciencias de la Educación han obtenido dicho reconocimiento. Esto supone un logro importante para esta área, ya que de las revistas incluidas en el bloque de Ciencias Sociales, las de educación son, con bastante diferencia, las que más evaluaciones positivas han conseguido.

Paralelamente, en los últimos años ha aumentado la presencia de las revistas españolas de educación en los listados del Journal Citation Reports, SCOPUS y ERIH, entre otros. Ahora bien, este éxito no ha supuesto una difusión científica equivalente de todas las especialidades educativas. La pregunta inicial que esta cuestión suscita es la situación particular de la gerontología educativa.

\section{OBJETIVOS}

El presente artículo analiza la incidencia de la gerontología educativa en ocho de las principales revistas científicas sobre educación que se publican en España. Para ello, se han planteado los siguientes objetivos:

- Ofrecer una visión diacrónica de la productividad científica sobre gerontología educativa durante el periodo 2000-2014.

- Describir un panorama de la gerontología educativa en España a través del análisis cuantitativo y cualitativo de una serie de variables.

- Trazar los rasgos característicos de este campo de conocimiento y esbozar algunas propuestas para avanzar en su desarrollo.

\section{METOdOLOGÍA}

La primera fase consistió en la selección de las revistas y, a continuación, se detectaron y clasificaron los artículos en función de su contenido, año de publicación, revista, titulación e institución de procedencia de los autores. Esta primera tarea proporcionó un acercamiento al desarrollo temporal de la producción científica en este campo, a los intereses de las diferentes revistas, así como a las instituciones más comprometidas con esta especialidad educativa. En la segunda fase, de carácter cualitativo, se realizó un análisis de contenido de los textos para obtener información sobre los intereses de los autores y las tendencias en la investigación en gerontología educativa.

Las revistas seleccionadas para este estudio son las recogidas en la categoría "Education \& Educational Research" del Journal Citation Report (2012) de Thomson Reuters. El motivo de esta selección que limita notablemente la muestra es que, anualmente, en los criterios pertenecientes al "Campo de las Ciencias Sociales, Políticas, del Comportamiento y de la Educación" por los que se evalúa la actividad investigadora del profesorado universitario en España, las publicaciones que se valoran preferentemente son en primer lugar: "Los artículos publicados en revistas de reconocida valía, aceptándose como tales las incluidas en los listados por ámbitos científicos del "Journal Citation Reports (Social Sciences Edition)" y "Journal Citation Reports (Science Edition)" del "Web of Science»". 
De ahí que estas revistas sean las más "buscadas" por el profesorado universitario para la publicación de sus trabajos académicos y, por tanto, las que finalmente recogen los artículos de mayor impacto.

La revista de educación más consolidada en esta base de datos es la Revista Española de Pedagogía, con una presencia sostenida desde el año 2007, las otras son consideradas revistas de reconocida valía y están incluídas en SCOPUS, en DICE-CINDOC, y algunas en ERIH, en el período analizado. Estas revistas son:

- Revista Española de Pedagogía

- Comunicar. Revista Científica Iberoamericana de Comunicación y Educación

- Revista de Educación

- Porta Linguarum

- Enseñanza de las Ciencias

- Cultura y Educación

- ESE- Estudios sobre Educación

- Educación XXI

El período elegido para el análisis son quince años, entre 2000 y 2014 (ambos inclusive). Ello nos va a dar una idea acerca de los intereses que han predominado dentro de la comunidad científica durante el período estudiado y nos facilitará una perspectiva de la evolución de la gerontología educativa en España en los últimos tres lustros.

Una vez seleccionada la muestra, se realizó una búsqueda -en los artículos de cada una de las revistas- de los siguientes términos: gerontología, vejez, envejecimiento, intergeneracional, personas mayores, abuelo, nieto. Para la selección de un artículo debía figurar al menos uno de dichos términos en el título, palabras clave o resumen.

El número de artículos sobre educación y personas mayores encontrados en dichas revistas durante los quince años analizados es de 17. En la tabla $1^{4}$ se especifican los artículos encontrados en las diferentes revistas y su año de publicación.

Para realizar el análisis de cada uno de los artículos se han definido una serie de variables: tipo, palabras clave/descriptores, multidisciplinariedad, género y aproximación holística al envejecimiento. Estas variables nos permitirán clarificar las principales características de la literatura científica seleccionada.

Tabla 1: Artículos sobre gerontología educativa publicados en revistas de educación en el período 2000-2014.

\begin{tabular}{|c|c|c|c|c|c|c|}
\hline & $\begin{array}{c}\text { Revista } \\
\text { Española de } \\
\text { Pedagogía }\end{array}$ & Comunicar & $\begin{array}{c}\text { Revista de } \\
\text { Educación }\end{array}$ & $\begin{array}{c}\text { Estudios } \\
\text { Sobre } \\
\text { Educación }\end{array}$ & $\begin{array}{c}\text { Educación } \\
\text { XXI }\end{array}$ & Totales \\
\hline $\mathbf{2 0 0 0}$ & & 1 & 1 & & & 2 \\
\hline $\mathbf{2 0 0 1}$ & & & 2 & & 1 & 3 \\
\hline $\mathbf{2 0 0 2}$ & & & & & & 0 \\
\hline
\end{tabular}

\footnotetext{
${ }^{4}$ Las revistas Cultura y Educación, Enseñanza de las Ciencias y Porta Linguarum no aparecen en la tabla porque no han publicado ningún artículo sobre gerontología educativa en el periodo analizado.
} 


\begin{tabular}{|c|c|c|c|c|c|c|}
\hline 2003 & 1 & & & & & 1 \\
\hline 2004 & & & & & & 0 \\
\hline 2005 & & 1 & 1 & & & 2 \\
\hline 2006 & & & & & & 0 \\
\hline 2007 & 1 & & & & & 1 \\
\hline 2008 & 1 & & 1 & & & 2 \\
\hline 2009 & & & & 1 & & 1 \\
\hline 2010 & & & & & & 0 \\
\hline 2011 & & 1 & & & & 1 \\
\hline 2012 & & 1 & & & & 1 \\
\hline 2013 & & & 1 & & & 1 \\
\hline 2014 & 0 & 2 & 0 & 0 & 0 & 2 \\
\hline Total & 3 & 6 & 6 & 1 & 1 & 17 \\
\hline
\end{tabular}

Fuente: Elaboración propia

Con respecto a la primera de las variables, y siguiendo la nomenclatura científica utilizada en otros estudios similares (Flores et al., 2010), los artículos se han clasificado en los siguientes tipos: teórico, práctico y empírico.

"El artículo clasificado como teórico es aquel que desarrolla conceptos, teorías, historia, desde un punto de vista conceptual y de paradigma. Incluimos como artículo práctico las experiencias y/o aplicaciones que se derivan del texto analizado, incluyendo a veces anexos que le dan esa vertiente aplicada. Finalmente, clasificamos los artículos en el tipo empírico, cuando éstos siguen las normas del proceso científico: hipótesis, objetivos, procedimiento, muestra, conclusiones" (Flores et al. 2010, p. 52).

En el caso de los artículos empíricos se diferenciarán los que sean fruto de investigaciones financiadas.

En cuanto a las palabras clave se determinará las que han sido más utilizadas y, posteriormente, se realizará una agrupación de las mismas que nos permita determinar el campo de estudio o de intervención.

El análisis de los autores de los artículos incluye la dimensión multidisciplinar, que hará referencia a la titulación universitaria de cada uno de los autores. Asimismo, se tendrán en cuenta la cuestión del género y el centro de trabajo de los autores.

Por último, otra de las variables que interesaba para este estudio era comprobar si los artículos analizados contemplan una aproximación holística al envejecimiento, es decir, si se tiene en cuenta el contexto, la diversidad así como la dimensión de una percepción longitudinal.

\section{RESULTADOS}

El primer dato que llama la atención es el escaso número de artículos sobre vejez y educación publicados en las revistas de educación analizadas, llegando incluso, en algunos años (2002, 2004, 2006 y 2010), a no publicar ninguno. Frente a ello, y como se puede apreciar en la Figura 1, el año en el que aparecen más artículos sobre esta temática es en 2001. 


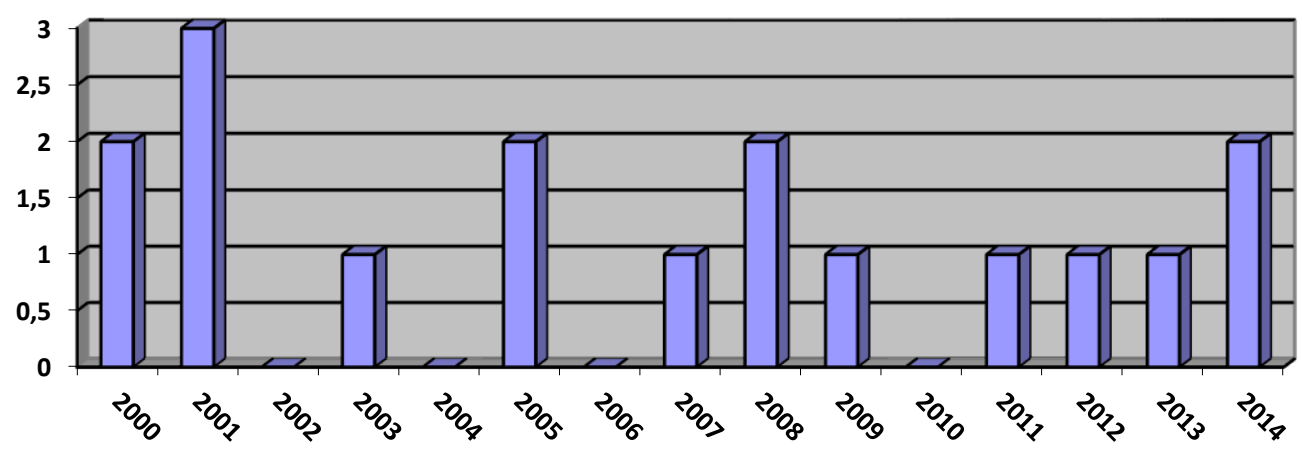

Figura 1: Productividad diacrónica

Una posible explicación de esta situación podría venir del hecho de la cercanía de dichas fechas con el año 1999, designado "Año Internacional de las Personas Mayores" por la Asamblea General de Naciones Unidas en la resolución 47/5 de 1992, o al año 2002 en el que se celebró la II Asamblea Mundial sobre el Envejecimiento en Madrid, también convocada por Naciones Unidas. No obstante, según esto, este último acto tendría que haber alentado, a su vez, la elaboración de nuevos artículos en los años siguientes pero únicamente encontramos uno publicado en 2003. Por tanto, se podría deducir que las declaraciones y celebraciones que Naciones Unidas realiza sobre el envejecimiento y las personas mayores no repercuten en la mayor o menor publicación de artículos, si bien los artículos publicados sí recogen todo el trabajo de Naciones Unidas en torno a este tema.

En lo que se refiere a las revistas, como se puede apreciar en la tabla 1, dos de ellas, la Revista de Educación, publicación del Ministerio de Educación, y la Revista Comunicar, son las que más artículos ( 6 en ambos casos) han publicado en los números analizados. Si bien este dato debe relativizarse ya que estas revistas publican un mayor número de artículos que algunas de las otras revistas analizadas. Por poner un ejemplo, frente a los cinco artículos publicados por la revista Educación XX1 en el año 2001, la Revista de Educación publicó cuatro números con un total de 86 artículos, de aquí que el hecho de que en ese año la Revista de Educación publicara el doble de artículos sobre gerontología educativa que la revista Educación XX1 no se puede considerar significativo. Más bien al contrario, que la revista de la UNED publicara en ese año un artículo sobre este tema es el dato realmente relevante. Sin embargo, la finalidad de este análisis no es tanto la comparación entre revistas sino el interés global mostrado hacia la gerontología educativa entre los años 2000 y 2014 por las revistas de educación españolas incluidas en el Journal Citation Report, por lo que este trabajo no se extiende más en esta cuestión.

El resto de revistas analizadas apenas tienen entre 1 y 3 artículos sobre este ámbito y tres de las revistas no han publicado ninguno. Entre estas últimas, llama la atención el caso de la revista Cultura y Educación ya que contrasta con la temática de esta revista. La descripción que encontramos en la web de Cultura y Educación es la siguiente: "es una revista internacional que admite trabajos de reflexión, de investigación educativa y experiencias didácticas debidamente evaluadas sobre los fenómenos educativos escolares, familiares y sociales culturalmente situados a lo largo de todas las etapas vitales" (la cursiva es nuestra). Al hacer explícito que los trabajos que pueden ser presentados a esta revista pueden estar referidos a cualquier etapa de la vida, se está 
incluyendo la vejez, por tanto, se echa de menos la publicación de artículos que atiendan a esta cuestión.

Sobre el aspecto de multidisciplinariedad, el análisis de los artículos muestra que de los 30 autores, 15 son doctores en Educación y, de éstos, tres han publicado dos artículos en este período, por lo que en esta muestra tenemos 12 doctores en Educación. Ahora bien, si tenemos en cuenta los textos escritos por estos 12 autores, encontramos que, o bien escriben solos (en su mayoría, ya que 6 de los 7 artículos con un solo autor son de estos doctores), o bien, tienden a escribir con otros doctores de su misma especialidad. En este último caso, los coautores son compañeros de Facultad y de Departamento en dos de los casos.

Casi la mitad de los artículos de la muestra han sido elaborados por un solo autor (4 de hombres y 3 de mujeres). De los 7 autores, uno de ellos es profesor de la Facultad de Sociología de la U. de La Coruña, otro es profesor de la Facultad de Humanidades y Ciencias de la Comunicación de la U. San Pablo CEU de Madrid y el resto son, en su mayoría, profesores de las facultades de educación de diversas universidades: Universidad Complutense de Madrid, Universidad Pontificia de Salamanca, Universidad de Salamanca, Universidad de Cádiz y Universidad de las Islas Baleares.

Otros 7 artículos han sido escritos por dos autores y en los últimos años (2011, 2012 y 2013) se publicaron tres artículos con tres autores. De estos 10 artículos con coautores, seis son de compañeros de Facultad, de lo que se deriva una escasa colaboración no sólo entre universidades sino también entre facultades dentro de la misma universidad. En uno de los casos el artículo está escrito por una doctoranda y su directora de tesis. Destaca, asimismo, que sólo el artículo de 2011 ha sido publicado en colaboración con una profesora de una universidad de Portugal, por lo que el grado de internacionalización es realmente poco significativo en estas publicaciones.

En la Figura 2 se ha recogido, además de la productividad personal, a través del apartado "autores" (se han considerado todos los firmantes de cada artículo), la productividad institucional (se asignó a cada universidad los artículos de sus respectivos profesores o investigadores). En este último apartado, destaca el hecho de que sólo uno de los autores es profesor de un Centro de Educación Secundaria y, en este caso, la autoría se comparte con una profesora universitaria. Los resultados muestran también un notable equilibrio en la producción de las diversas universidades, si bien destacan cinco de ellas especialmente por la producción de los autores (Universidad de Burgos, Universidade de Vigo, Universidad de Salamanca, Universitat de les Illes Balears y Universidad de Oviedo).

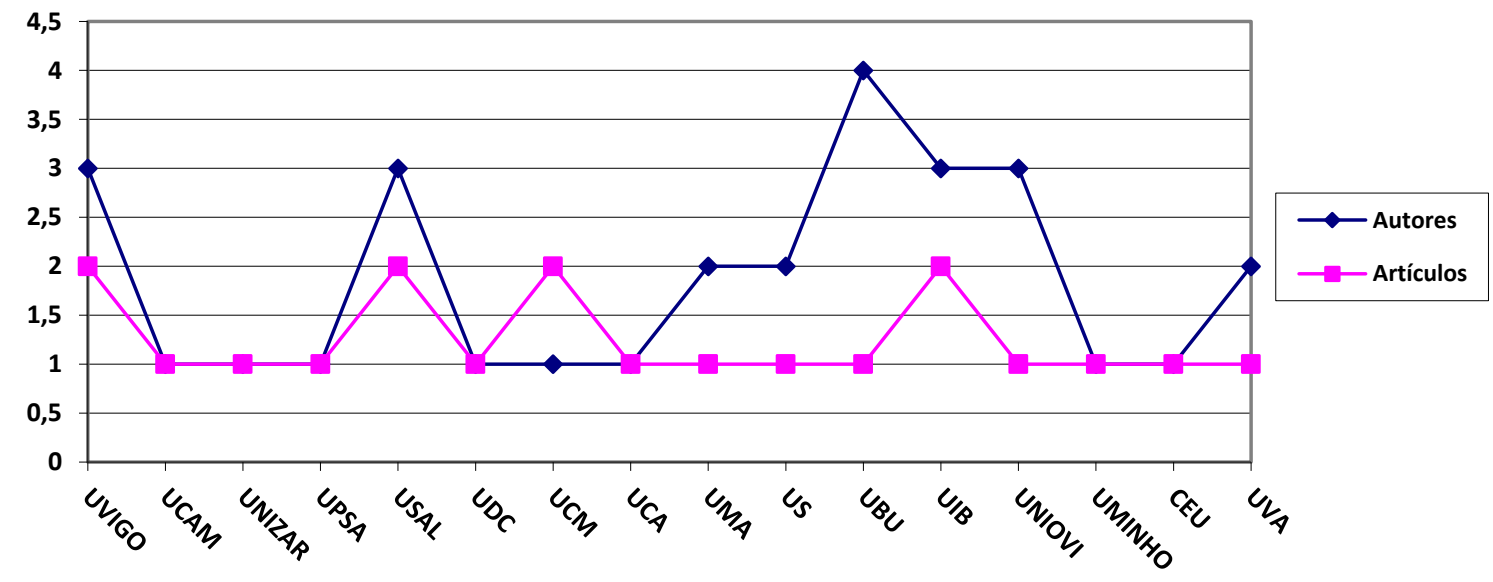

Figura 2: Producción institucional 
La distribución por revista, año, género y titulación de los autores quedan reflejados en la tabla 2.

Tabla 2. Artículos sobre gerontología educativa publicados en revistas de educación en el período 2000-2014 por género y titulación de los autores.

\begin{tabular}{|c|c|c|c|}
\hline \multirow{2}{*}{ Revista/Año/№ } & \multicolumn{2}{|c|}{ Autores } & \multirow{2}{*}{ Titulación } \\
\hline & Mujer & Hombre & \\
\hline $\begin{array}{l}\text { Revista de Educación/2013/ } \\
\text { Extraordinario }\end{array}$ & 3 & & $\begin{array}{l}\text { Licenciada y Dra. En Educación Física; Licenciada en } \\
\text { Educación Física; Licenciada y Dra. en Educación Física }\end{array}$ \\
\hline $\begin{array}{l}\text { Revista de } \\
\text { Educación/2008/346 }\end{array}$ & 1 & & Dra. en Ciencias de la Educación \\
\hline $\begin{array}{l}\text { Revista de Educación/ } \\
\text { 2005/338 }\end{array}$ & & 2 & $\begin{array}{l}\text { Dr. en Ciencias de la Educación; Licenciado y Dr. en } \\
\text { Filosofía y Ciencias de la Educación }\end{array}$ \\
\hline $\begin{array}{l}\text { Revista de } \\
\text { Educación/2001/325 }\end{array}$ & & 1 & Licenciado y Doctor en Ciencias Políticas y Sociología \\
\hline $\begin{array}{l}\text { Revista de } \\
\text { Educación/2001/324 }\end{array}$ & 1 & & Licenciada y Dra. en Ciencias de la Educación \\
\hline $\begin{array}{l}\text { Revista de } \\
\text { Educación/2000/323 }\end{array}$ & & 1 & Licenciado y Doctor en Ciencias de la Educación \\
\hline Comunicar/2014/42 & 2 & & $\begin{array}{l}\text { Licenciada en Publicidad y Relaciones Públicas y } \\
\text { Doctora en Ciencias de la Información; Licenciada } \\
\text { en Publicidad y Relaciones Públicas y Doctora en } \\
\text { Publicidad. }\end{array}$ \\
\hline Comunicar/2014/42 & & 1 & $\begin{array}{l}\text { Licenciado en Derecho y Doctor en Ciencias de la } \\
\text { Información }\end{array}$ \\
\hline Comunicar/2012/39 & 2 & 1 & $\begin{array}{l}\text { Maestra, Licenciada en Pedagogía y Dra. En Ciencias } \\
\text { de la Educación; Licenciada y Dra. En Filosofía y CC. De } \\
\text { la Educación; Licenciado y Dr. En CC. De la Información y } \\
\text { Licenciado en Filosofía y CC. De la Educación. }\end{array}$ \\
\hline Comunicar/2011/37 & 2 & 1 & $\begin{array}{l}\text { Dr. en Psicología; Dra. en Ciencias de la Información; } \\
\text { Licenciada en Historia y Pedagogía y Dra. en Psicología. }\end{array}$ \\
\hline $\begin{array}{l}\text { Comunicar/ } \\
2005 / 25\end{array}$ & & 2 & $\begin{array}{l}\text { Licenciado en CC. de la Comunicación; Licenciado en } \\
\text { CC. de la Comunicación. Ambos son doctorandos en } \\
\text { CC. de la Comunicación. }\end{array}$ \\
\hline Comunicar/2000/15 & & 1 & $\begin{array}{l}\text { Licenciado en Ciencias Químicas y Doctor en Ciencias de la } \\
\text { Educación }\end{array}$ \\
\hline $\begin{array}{l}\text { Estudios sobre Educación } \\
/ 2009 / 17\end{array}$ & 2 & & $\begin{array}{l}\text { Dra. en Ciencias de la Educación; Dra. en Ciencias de la } \\
\text { Educación }\end{array}$ \\
\hline Educación XXI/2001/4 & 1 & 1 & $\begin{array}{l}\text { Licenciada y Dra. en Ciencias de la Educación; } \\
\text { Licenciado y Dr. en Filosofía }\end{array}$ \\
\hline $\begin{array}{l}\text { Revista Española } \\
\text { de Pedagogía/2008/239 }\end{array}$ & 1 & 1 & $\begin{array}{l}\text { Dr. En Psicología; Licenciada en Psicología y en } \\
\text { Pedagogía y Dra. en Ciencias de la Educación }\end{array}$ \\
\hline $\begin{array}{l}\text { Revista Española de } \\
\text { Pedagogía /2007/237 }\end{array}$ & 1 & 1 & $\begin{array}{l}\text { Licenciada en Psicología y Dra. en Ciencias de la } \\
\text { Educación; Licenciado y Dr. en Ciencias de la Educación }\end{array}$ \\
\hline $\begin{array}{l}\text { Revista Española } \\
\text { de Pedagogía/2003/226 }\end{array}$ & 1 & & Dra. en Ciencias de la Educación \\
\hline
\end{tabular}

A la vista de los datos aportados en la tabla 2, se puede afirmar que el grado de multidisciplinariedad en los artículos analizados es muy bajo ya que, de los 10 artículos escritos en colaboración, 3 han sido escritos por doctores en Ciencias de la Educación, uno por dos doctores en CC. de la Educación y un licenciado en CC. de la Educación, uno por dos doctoras en Educación Física y una licenciada en Educación Física, uno por compañeros de estudios (es el caso de dos doctorandos en Ciencias de la Comunicación), uno por una doctora en Psicología y una licenciada 
en Psicología y uno por dos licenciadas en Publicidad y Relaciones Públicas (una de ellas doctora en Ciencias de la Información y otra en Publicidad). Sólo dos de los artículos han sido escritos por licenciados y doctores en diferentes materias: Ciencias de la Educación y Filosofía en uno de ellos y Psicología y Ciencias de la Información en el otro.

Este bajo grado de multidisciplinariedad constituye una tendencia general en la investigación sobre envejecimiento en España. Como apunta Rodríguez $(2011,9)$, "Siguen dominando todavía las investigaciones 'disciplinares' sobre envejecimiento con una clara separación entre las ciencias 'bio' y ciencias sociales y psicología”.

En lo que respecta al tipo de artículo (teórico, práctico o empírico), en la tabla 3 se exponen los tipos encontrados en los diferentes artículos. El análisis de esta variable nos lleva a una conclusión llamativa y es la distribución bastante equilibrada entre los tres tipos. En primer lugar, hay seis artículos empíricos y de ellos sólo uno refiere que los datos allí recogidos son parte de una investigación financiada por el Ministerio de Ciencia y Tecnología (este dato es coherente con la información obtenida anteriormente sobre el bajo número de proyectos de investigación financiados en este período). En segundo lugar, hay cuatro artículos que podríamos calificar como teóricos. Y por último, siete artículos analizados podrían calificarse como teórico/prácticos, ya que la mayor parte de las páginas se dedican a reflexión, descripción y exposición de teorías y modelos pero en la última parte se incluyen propuestas de cursos y otras intervenciones socioeducativas y se alude a experiencias e investigaciones realizadas, ya sea por los propios autores o por otros.

Tabla 3. Análisis en función del tipo de artículo.

\begin{tabular}{|c|c|}
\hline Revista/Año/Número & Tipo de artículo \\
\hline $\begin{array}{l}\text { Rvta. De } \\
\text { Educación/2013/Extraordinario }\end{array}$ & Empírico \\
\hline Rvta. de Educación/2008/346 & Teórico \\
\hline Rvta. de Educación/2005/338 & $\begin{array}{l}\text { Práctico: experiencia de los programas universitarios para } \\
\text { mayores en Galicia }\end{array}$ \\
\hline Rvta. de Educación/2001/325 & Teórico \\
\hline Rvta. de Educación/2001/324 & Teórico \\
\hline Rvta. de Educación/2000/323 & Teórico \\
\hline Rvta. Comunicar/2014/42 & $\begin{array}{l}\text { Práctico: incluye propuestas metodológicas para el diseño de } \\
\text { programas de e-inclusión de personas mayores }\end{array}$ \\
\hline Rvta. Comunicar/2014/42 & Empírico \\
\hline Rvta. Comunicar/2012/39 & Empírico \\
\hline Rvta. Comunicar/2011/37 & Empírico \\
\hline Rvta. Comunicar/2005/25 & Empírico \\
\hline Rvta. Comunicar/2000/15 & $\begin{array}{l}\text { Práctico: incluye la propuesta de un curso sobre el uso de las TIC } \\
\text { por personas mayores }\end{array}$ \\
\hline $\begin{array}{l}\text { Rvta. Estudios sobre } \\
\text { Educación/2000/15 }\end{array}$ & Empírico (financiado por el Ministerio de Ciencia y Tecnología) \\
\hline Rvta. Educación XXI/2001/4 & $\begin{array}{l}\text { Práctico: el artículo acaba ofreciendo temas para un curso de } \\
\text { educación para la salud. }\end{array}$ \\
\hline
\end{tabular}




\begin{tabular}{|l|l|}
\hline $\begin{array}{l}\text { Rvta. Española de } \\
\text { Pedagogía/2008/239 }\end{array}$ & $\begin{array}{l}\text { Práctico: la mayor parte del artículo es teórico pero al final se } \\
\text { incluye un resumen de una investigación realizada tres años } \\
\text { antes por los autores. }\end{array}$ \\
\hline $\begin{array}{l}\text { Rvta. Española de } \\
\text { Pedagogía/2007/237 }\end{array}$ & $\begin{array}{l}\text { Teórico, aunque se incluye unas indicaciones para la } \\
\text { intervención socioeducativa. }\end{array}$ \\
\hline $\begin{array}{l}\text { Rvta. Española de } \\
\text { Pedagogía/2003/226 }\end{array}$ & $\begin{array}{l}\text { Teórico. Además, hace alusión a diversas experiencias de } \\
\text { pedagogía museística }\end{array}$ \\
\hline
\end{tabular}

Fuente: Elaboración propia

En cuanto a los descriptores, en cinco de los artículos no figuran. Se trata, en su mayoría, de artículos del año 2005 o anteriores, a excepción de un artículo de la Revista Educación XXI que, pese a que el artículo analizado es del año 2001, sí tiene descriptores. Hay que decir que en la actualidad todas las revistas, en sus normas de publicación/edición, exigen palabras clave o descriptores, tanto en español como en inglés (entre 4 y 10). Y algunas de ellas tienen además otras indicaciones:

- La Revista Comunicar, cuenta con un Thesaurus de palabras clave con los términos más utilizados en las temáticas de la revista aunque también se recomienda el uso del Thesauro de la UNESCO

- La Revista Educación XXI pide que sean extraídas del Tesauro Europeo de Educación, http://eurydice.org/portal/page/portal/Eurydice/TEE o bien del Tesauro de ERIC <http:www.eric.ed.gov>

- Estudios sobre Educación sugiere que se utilicen el Psychology Glossary accesible en http://www.apa.org/ o el Tesauro Europeo de la Educación, accesible en http://www.eurydice.org.

- Revista de Educación sugiere el Tesauro de Eric.

Una vez analizados los descriptores, se comprueba que sólo uno de ellos coincide en siete artículos (personas mayores/mayores) y otros tres descriptores (educación gerontológica, gerontología educativa y calidad de vida) en dos artículos. El resto de descriptores sólo son utilizados una vez.

La diversidad de términos y temas encontrados refleja la amplitud de intereses de los autores. Ello ha propiciado la elaboración de una clasificación que los agrupa en función de su proximidad a determinados campos de estudio o de intervención y que muestra, de una manera más gráfica, las aportaciones derivadas de la reflexión, experiencia, estudio e investigación en los quince años analizados. La clasificación realizada es la siguiente:

- Temática general: educación en la vejez, educación gerontológica, gerontología educativa, educación permanente, educación, envejecimiento y personas mayores.

- Subtemas (campos de estudio o de intervención): Conceptos/modelos, políticas, salud, ocio, tecnologías y relaciones intergeneracionales.

- Conceptos/Modelos: práctica reflexiva, educación intergeneracional, publicidad, imágenes sociales, reflexividad crítica, modelo actancial

- Políticas: Planes gerontológicos, política social y políticas públicas. 
- Salud: recursos sociosanitarios, educación para la salud, autocuidado, vida saludable, salud y bienestar, educación física

- Ocio: recursos de ocio, espacios para mayores, ciudad educadora, televisión, ficción televisiva, grupos de debate, animación sociocultural, ocio y tiempo libre y museo.

- Tecnologías: Internet, TIC, cultura digital, alfabetización mediática, competencia mediática, brecha digital, televisión en España y ficción televisiva.

- Relaciones intergeneracionales: nieto favorito, rol del abuelo.

Por último, el análisis sobre la aproximación holística al envejecimiento nos revela que gran parte de los artículos hacen referencia al contexto (en dos de ellos se analizan diferentes contextos y espacios pero en la mayoría de artículos simplemente se hace alusiones generales) y a la diversidad de las personas mayores, afirmándose que se trata de un colectivo plural. Sin embargo, la dimensión "a lo largo de la vida" apenas es trabajada. Sólo en dos de ellos podríamos considerarla cuando se reflexiona sobre modelos que abordan el tema de la vejez como continuidad. Por otra parte, ninguno de los artículos recoge estudios longitudinales.

\section{CONCLUSIONES Y ALGUNAS PROPUESTAS}

Los datos aquí analizados no constituyen una imagen exhaustiva de la producción científica española en materia de gerontología educativa pero su relevancia se deriva de la alta visibilidad e impacto de la muestra de revistas seleccionadas. En todo caso, el análisis de la investigación publicada en las revistas científicas ofrece datos de interés sobre las áreas de investigación que se consideran relevantes y que, por tanto, se estima deben ser objeto de publicación (Buboltz et al., 1999; Eby et al. , 2005). Al mismo tiempo, también las revistas influyen en la formación y evolución de ámbitos científicos, como muestra, en el caso que nos ocupa -la gerontología-, la investigación realizada por Mercer y Carter (2012).

En concreto, en este estudio se planteaba si las publicaciones científicas españolas se han hecho eco del fenómeno del envejecimiento de la población y han contribuido a la construcción de la gerontología educativa. Se ha podido comprobar que los artículos publicados sobre este ámbito, en la muestra analizada, son escasos y limitados, especialmente los artículos derivados de investigaciones financiadas. No obstante, sería interesante conocer, para una interpretación más adecuada de estos datos, el número de artículos sobre gerontología educativa enviados a las revistas analizadas en el período seleccionado, ¿el número de publicaciones no es mayor porque los especialistas en el tema no han realizado más aportaciones? Lamentablemente, las cifras que publican algunas revistas sobre los artículos recibidos sólo están referidas a las secciones a las que son enviados, por lo que resulta imposible conocer la temática de los artículos rechazados.

Más allá de esta situación, la escasez de los artículos publicados revela la necesidad de un apoyo más afianzado a las investigaciones en esta área y una mayor difusión científica que permita y propicie una construcción más sólida de este campo de conocimiento. A este respecto, el estudio de Infeld y Adams (2013) aporta una visión novedosa atendiendo al generalizado uso de Internet para la búsqueda de información sobre cualquier tema. Dichos autores apelan a una tarea ineludible, la de la mejora de Wikipedia en su cobertura sobre temas gerontológicos, ya que detectaron notables carencias en este ámbito. 
El desarrollo de la gerontología educativa es necesario para poder progresar tanto en su conocimiento como en su aplicación. La acuciante necesidad del aprendizaje a lo largo de la vida en una sociedad en constantes cambios que nos afectan a todos a través de cada una de las actividades diarias hace que debamos replantearnos las múltiples cuestiones referidas a las personas mayores y su relación con el ámbito educativo, así como la formación de profesionales capacitados para trabajar con los mayores (Bergman, Erickson y Simons, 2014). En especial, sería necesario llevar a cabo investigaciones longitudinales que den respuesta a los múltiples interrogantes que aún hoy, en una sociedad inmersa en un profundo proceso de envejecimiento, siguen pendientes de ser resueltos.

En lo referente al proceso de construcción de la gerontología educativa como ámbito de conocimiento, -o quizá podríamos decir "reconstrucción", dado que en los últimos años, aunque no se hayan publicado un número suficiente de artículos sí se ha publicado un número algo más considerable de monografías- será necesario revisar y repensar las nociones, los conceptos y los modelos que han fundamentado el discurso educativo en la vejez. Algunos de los artículos analizados trabajan estas cuestiones pero habría que persistir más en ello pues siguen siendo iniciativas aisladas.

En este proceso sería oportuno realizar, asimismo, un profundo estudio de la diversidad de praxis educativas que se están llevando a cabo en los centros e instituciones donde se trabaja con personas mayores y analizar los planteamientos metodológicos que se están utilizando. Igualmente, sería pertinente determinar el modelo de educador que requieren las nuevas generaciones de mayores.

Otro de los objetivos de este trabajo era estudiar la multidisciplinariedad en las publicaciones seleccionadas. Los resultados del análisis muestran que el enfoque multidisciplinar está prácticamente ausente. Esto supone una barrera para la adecuada implementación de la perspectiva del aprendizaje a lo largo de la vida que requiere tener en cuenta al individuo en sus diversas facetas así como los contextos y las circunstancias en las que se llevan a cabo las experiencias educativas. La multidisciplinariedad ha sido considerada como una de las prioridades de investigación sobre envejecimiento en la colaboración europea, sin embargo, como reconoce Rodríguez (2011, p. 9), en España existe una tendencia hacia las investigaciones "disciplinares" sobre envejecimiento. Por tanto, este es otro de los retos que tendremos que afrontar en los próximos años, tanto desde el área de humanidades y ciencias sociales, y en lo que aquí atañe desde el ámbito de la educación, como desde las áreas de ciencias "bio" y tecnológicas.

A raíz del análisis llevado a cabo, surgió otra cuestión a debatir, el papel de los tesauros o catálogos de palabras clave que las revistas científicas suelen sugerir, o algunas incluso llegan a exigir. La realidad es que los autores no siempre los utilizan ${ }^{5}$ y los descriptores o palabras clave de los artículos suelen ser fruto más de la capacidad de concreción y la creatividad de los autores que del uso de un lenguaje estandarizado. Este hecho sumado a la diversidad de catálogos existentes y a la ausencia de palabras clave en algunos artículos (como se ha podido comprobar especialmente en la primera parte del período analizado) impide un análisis válido desde un criterio de estandarización y control. Sin embargo, esta situación también tiene una ventaja y es que la falta de una mayor inclusión de términos relacionados con la vejez en los catálogos de

\footnotetext{
${ }^{5}$ Posiblemente esto seguirá ocurriendo hasta que los informes o protocolos de evaluación de manuscritos lo incluyan como un elemento más de la evaluación, lo que en la actualidad no es habitual.
} 
educación se suple con una riqueza de vocablos y expresiones que ayudan a construir un perfil bastante aproximado del contenido de los artículos. En el caso que nos ocupa, se ha comprobado que las temáticas abordadas en los artículos están relacionadas con conceptos/modelos, políticas, salud, ocio, tecnologías y relaciones intergeneracionales. ¿Qué perfil se hubiera construido si en estos artículos sólo se hubiera utilizado uno de los catálogos sugeridos por las revistas? ¿Nos habría ofrecido más pistas sobre el contenido de los artículos o el resultado hubiera sido más superficial?

La alusión a los subtemas tratados en los artículos analizados conduce, de nuevo, al interés hacia el trabajo multidisciplinar. Aquí surgen cuestiones tales como: ¿Sería preferible que un artículo sobre gerontología educativa y salud lo escribiera un especialista del ámbito educativo junto a otro del ámbito sanitario, tal vez un geriatra? ¿O que lo escribieran sólo especialistas de uno de los ámbitos? ¿Qué ventajas e inconvenientes tendría cada una de las opciones?

Las cuestiones expuestas anteriormente demandan una condición de la que también adolece la investigación actual y que está suponiendo un problema en el avance de la gerontología educativa, y es la escasez de trabajos en los que se cuenta con la opinión de las personas mayores. En este sentido se manifestaba el Comité de las Regiones (2012, p. 47), en su dictamen sobre el envejecimiento activo, al insistir "en la importancia de consultar e involucrar activamente a las personas mayores junto con sus cuidadores en la descripción de sus necesidades, el desarrollo de soluciones y la evaluación de sus logros". En el ámbito que aquí nos atañe, es fundamental preguntarles sobre el aprendizaje y la educación, qué quieren y qué necesitan aprender, cómo y por qué quieren aprender y qué significado le dan al aprendizaje. Incluso se ha llegado a discutir sobre la necesidad de un nuevo paradigma de investigación que sitúe a los mayores en el centro del debate y los incorpore en el proceso de investigación (Boulton-Lewis, 2010). Esta misma laguna, como hemos podido comprobar en la literatura analizada, se encuentra también en la investigación realizada en España. En el estudio realizado sólo encontramos un caso en el que se ha involucrado a una persona mayor, ya que en realidad el artículo publicado muestra los resultados de su tesis doctoral. En este caso, por tanto, ha sido una persona mayor la que ha realizado una investigación sobre un tema gerontológico. Esta excepción debería constituir un aliciente para que en un futuro cercano los mayores ocupen un papel central en la investigación gerontológica.

\section{REFERENCIAS}

1. BERGMAN, E., ERICKSON, M. A. Y SIMONS, J. Attracting and Training Tomorrow's Gerontologists: What Drives Student Interest in Aging? Educational Gerontology, v. 40, n. 3, p. 172-185, 2014.

2. BOULTON-LEWIS, G. Education and Learning for the Elderly: Why, How, What". Educational Gerontology, 36, p. 213-228, 2010.

3. BUBOLTZ, W. C., MILLER, M. Y WILLIAMS, D. J. Content analysis of research in the "Journal of Counseling Psychology". Journal of Counseling Psychology, v. 46, n. 4, p. 496-503, 1999.

4. COMISIÓN EUROPEA. Propuesta de decisión del Parlamento Europeo y del Consejo sobre el Año Europeo del Envejecimiento Activo (2012). COM (2010) 462 final, 2010.

5. COMISIÓN EUROPEA. Informe de la Comisión al Parlamento Europeo, al Consejo, al Comité Económico y Social Europeo y al Comité de las Regiones sobre la puesta en práctica, los resultados y la evaluación general del Año Europeo del Envejecimiento Activo y de la 
Solidaridad Intergeneracional (2012). COM(2014) 562 final, 2014.

6. COMITÉ DE LAS REGIONES. Dictamen del Comité de las Regiones sobre el envejecimiento activo: Innovación, salud inteligente y mejores vidas. Diario Oficial de la Unión Europea, C 225/05, 2012.

7. CONSEJO DE LA UNIÓN EUROPEA. Conclusiones del Consejo sobre el papel de la educación y de la formación en la aplicación de la Estrategia Europa 2020. Diario Oficial de la Unión Europea, C70/1, 2011.

8. EBY, L. T., CASPER, W. J., LOCKWOOD, A., BORDEAUX, C. Y BRINLEY, A. Work and family research in IO/OB: Content analysis and review of the literature (1980-2002). Journal of Vocational Behavior, v. 66, n. 1, p. 124-197, 2005.

9. FECYT. Propuesta de manual de ayuda a los investigadores españoles para la normalización del nombre de autores e instituciones en las publicaciones científicas, 2007 Disponible en: http://www.accesowok.fecyt.es/wp-content/uploads/2009/06/normalizacion_nombre_au tor.pdf

10. FLORES, R., GIL, J. M., CABALLER, A. Y MARTíNEZ, M. A. La orientación educativa en la "Revista Española de Orientación y Psicopedagogía". Un estudio diacrónico y sincrónico (1990-2006). Bordón, v. 62, n. 1, p. 49-59, 2010.

11. HERNÁNDEZ, F. Y MAQUILÓN, J. Indicadores de calidad de las revistas científicas y sistema de gestión editorial mediante OJS, Revista de Investigación Educativa, v. 28, n. 1, p. 13-29, 2010.

12. IMSERSO. Presentación y resumen del Libro Blanco del Envejecimiento Activo. Temas para el debate, Madrid, 2010. Disponible en: http://www.imserso.es/InterPresent1/groups/im serso/documents/binario/extlbenvact20110126.pdf

13. INFELD, D. L. Y ADAMS, W. C. Using the Internet for Gerontology Education: Assessing and Improving Wikipedia. Educational Gerontology, v. 30, n. 10, p. 707-716, 2013.

14. INSTITUTO NACIONAL DE ESTADÍSTICA. Panorámica social de España, 1994. Madrid: Instituto Nacional de Estadística, 1995.

15. INSTITUTO NACIONAL DE ESTADÍSTICA. España en cifras 2014. Madrid: INE, 2014.

16. LUPPI, E. Education in old age: An exploratory study. International Journal of Lifelong Education v. 28, n. 2, p. 241-276, 2009.

17. MERCER, L. Y CARTER, L. Early Journals and Their Influences on the Development of Gerontology. Educational Gerontology, v. 38, p. 616-626, 2012.

18. RODRÍGUEZ, V. Futurage. Prioridades de la Investigación sobre Envejecimiento en Europa. Resultados de la consulta en España. Informes Portal Mayores 107, 2011. Disponible en: http://www.imsersomayores.csic.es/documentos/documentos/rodriguez-futurage-01.pdf

19. SCHOENMAECKERS, R. "Population ageing and its economic and financial implications". IN: R. Schoenmaeckers y I. Kotowska (coords.) Population ageing and its challenges to social policy, Strasbourg: Council of Europe Publishing, p. 27-116, 2005.

20. UNITED NATIONS. World Population Ageing 2009. New York, 2010.

21. UNITED NATIONS. World Population Prospects: The $\mathbf{2 0 1 2}$ Revision, Highlights and Advance Table., New York, 2013. Disponible en: http://esa.un.org/unpd/wpp/Documentation/pdf/ WPP2012_HIGHLIGHTS.pdf 